\title{
Ensino do médico residente em cirurgia geral: proposta de ferramenta didático-pedagógica
}

\author{
Teaching of the resident physician in general surgery: didactic and \\ pedagogical tool proposal
}

Igor Pereira de Carvalho Mestrando em Ensino em Ciências da Saúde e do Meio Ambiente Centro Universitário de Volta Redonda - UniFOA.

Volta Redonda, Rio de Janeiro - Brasil. igor.pereira.pec@hotmail.com

(iD) Adilson Pereira Doutorado em Filosofia Centro Universitário de Volta Redonda - UniFOA.

Volta Redonda, Rio de Janeiro - Brasil. adilsonfaetec@gmail.com

Ivanete da Rosa Silva de Oliveira Doutora em Educação Centro Universitário de Volta Redonda - UniFOA.

Volta Redonda, Rio de Janeiro - Brasil. ivanete.oliveira@foa.org.br

Resumo: Preparar médicos residentes em cirurgia geral e otimizar o domínio de competências é o contexto que moldou a situação-problema da presente investigação, cujo objetivo é avaliar como podemos obter melhores resultados na relação ensino-aprendizagem, implementada por proposta de ferramenta didático-pedagógica sob a forma de manual intitulado Guia do Residente em Cirurgia Geral, direcionado a preceptores e discentes que atuam nessa área. O referido manual é um produto educacional desenvolvido em Programa de Mestrado em Ensino em Ciências da Saúde e Meio Ambiente e foi avaliado, em primeiro momento, por médico cirurgião que atua como preceptor em residência médica e especialista nessa área, atendendo ao projeto piloto que coletou dados para subsidiar a elaboração de versão final a ser avaliada por juízes. Nessa fase da pesquisa, o método exploratório se apoiou na entrevista semiestruturada como instrumento de investigação, cujos resultados serão utilizados para a otimização da formação dos egressos nessa especialização.

Palavras-chave: cirurgia geral; ensino; internato e residência; materiais de ensino.

Abstract: Preparing medical residents in general surgery and optimizing the domain of competences is the context that shaped the problemsituation of the present investigation, whose objective is to evaluate how we can obtain better results in the teaching-learning relationship, implemented by the proposal of a didactic-pedagogical tool under the form of a manual entitled the General Surgery Resident's Guide, aimed at preceptors and students working in this area. The referred manual is an educational product developed in a Master's Program in Teaching in Health and Environmental Sciences and was evaluated, in the first instance, by a surgeon who acts as a preceptor in medical residency and a specialist in this area, attending the pilot project he collected data to support the preparation of the final version to be evaluated by judges. In this phase of the research, the exploratory method was supported by the semi-structured interview as a research instrument, the results of which will be used to optimize the training of graduates in this specialization.

Key-words: general surgery; teaching; internship and residency; teaching materials.

Cite como

\section{(ABNT NBR 6023:2018)}

CARVALHO, Igor Pereira de; PEREIRA, Adilson; OLIVEIRA, Ivanete da Rosa Silva de. Ensino do médico residente em cirurgia geral: proposta de ferramenta didático-pedagógica. Dialogia, São Paulo, n. 38, p. 1-13, e18959, maio/ago. 2021. Disponível em: https://doi.org/10.5585/38.2021.18959.

American Psychological Association (APA)

Carvalho, I. P. De., Pereira, A., Pereira, A., \& Oliveira, I. da. R. S. de. (2021, maio/ago.). Ensino do médico residente em cirurgia geral: proposta de ferramenta didático-pedagógica. Dialogia, São Paulo, 38, p. 1-13, e18959. https://doi.org/10.5585/38.2021.18959. 


\section{Introdução}

O presente estudo tem como objetivo avaliar um protótipo que consiste em uma ferramenta didático-pedagógica a ser implementada para contribuir com a formação de médicosresidentes em Cirurgia Geral, desenvolvida sob a forma de manual que procura otimizar o processo de aquisição de competências e habilidades necessárias à referida especialização. A presente pesquisa é resultado da segunda fase de estudo inicial, cuja motivação emergiu dos resultados de revisão integrativa elaborada para se obter dados relativos ao uso de materiais de ensino para médicos-residentes em cirurgia geral no Brasil, evidenciando-se lacunas no estado dessa questão.

Utilizou-se as bases de Souza et al. Integrative review: what is it? How to do it? (2010), para a implementação dessa primeira etapa da pesquisa, em que foram considerados os últimos 10 anos de estudos publicados e indexados às bases de dados PubMed e Scielo, tendo por critérios de inclusão: estudos publicados em língua inglesa e/ou portuguesa, disponíveis em versão eletrônica, que tratasse estritamente da temática ensino em cirurgia geral e materiais de ensino para médicos residentes, além de reflexões teóricas envolvendo esse assunto.

Os resultados encontrados foram sintetizados para melhor compreensão e ancoragem dessa etapa investigativa como tópico do suporte teórico relativo à problematização do ensino de competências e habilidades ao cirurgião geral. Os sujeitos aos quais se direcionam as preocupações da presente pesquisa são os médicos que atuam na preceptoria desta especialização, bem como os discentes que fazem, parte deste cotidiano.

Apresentam-se, portanto, síntese da base teórica utilizada para a fundamentação da pesquisa, a problematização do ensino na área investigada, o material didático-pedagógico intitulado Guia do Residente em Cirurgia Geral e a validação inicial do mesmo por especialista atuante na área de Cirurgia Geral. No intuito de se garantir o resguardo e amparo dos procedimentos adotados na aplicação da entrevista, a pesquisa recebeu parecer do Comitê de Ética em Pesquisa - COEPS/Centro Universitário de Volta Redonda - UniFOA/ Fundação Oswaldo Aranha, sob o CAAE: 42916819.8.0000.5237.

\section{Suporte Teórico}

\section{Considerações sobre a Residência Médica em Cirurgia Geral}

No final do século XIX, o cirurgião William Halsted (1852-1922) tencionou a criação do primeiro programa de residência médica no Hospital Johns Hopkins, criando assim, novo modelo de ensino-aprendizagem na área médica (COSTA et al., 2018; SANTOS et al., 2009 apud 
TONATTO FILHO et al., 2020). Tal ideia de organização foi importada pelo Brasil na década de 1950, inicialmente, no Rio de Janeiro, Hospital dos Servidores do Estado, e no Hospital de Clínicas em São Paulo (NACUL et al., 2015; TONATTO FILHO et al., 2020).

Considerando-se que a medicina é uma ciência em constante evolução, baseada no desenvolvimento técnico e incorporação de novas tecnologias. A Cirurgia geral, também em constante transformação, desenvolveu novas subespecialidades, incorporação de novas tecnologias, exigindo ao longo das décadas, novos métodos de treinamento e, consequentemente, modificação do processo de ensino-aprendizagem dos médicos-residentes (NACUL et al., 2015).

$\mathrm{O}$ autor compreende que a cirurgia deve ser objeto de treinamento desenvolvido paulatinamente, por etapas que ampliem a complexidade dos procedimentos, necessitando-se de tempo adequado para que o médico domine tais habilidades. Por isso, esse aprendizado deve ocorrer em serviço credenciado para tal atividade, com volume cirúrgico significativo, sob a supervisão de um preceptor (NACUL et al., 2015).

A Residência Médica é considerada um excelente método para formação em cirurgia, pois é baseada no treinamento em serviço, auxiliando assim a lapidação do conhecimento e aprimoramento de habilidades psicomotoras do pós-graduando (NACUL et al., 2015; COSTA et al., 2018).

Na esteira de convergência, Simpson (1972 apud COSTA et al., 2018) já indicava que o ensino de habilidades práticas é o componente central da educação cirúrgica, tanto de sua oferta na graduação, quanto na pós-graduação, pois a aprendizagem efetiva das habilidades psicomotoras é resultante da interação de diferentes aspectos, dentre os quais, o autor cita o meio ambiente (cenário de prática), a estruturação da prática, o diálogo professor/aluno e as estratégias pedagógicas.

No ano de 1977 foi emitido decreto para a regulamentação da Residência Médica e criação da Comissão Nacional de Residência Médica. Constituindo, assim, a residência médica sob a forma de especialização, como modalidade de pós-graduação voltada para médicos (BRASIL, 1977).

Art. 1o Este Decreto dispõe sobre a composição e a competência da Comissão Nacional de Residência Médica - CNRM, e sobre o exercício das funções de regulação, supervisão e avaliação das instituições que ofertam residência médica e de seus respectivos programas.

Art. 2o A CNRM é instância colegiada de caráter consultivo e deliberativo do Ministério da Educação e tem a finalidade de regular, supervisionar e avaliar as instituições e os programas de residência médica.

Parágrafo único. A regulação das instituições e dos programas de residência médica deverá considerar a necessidade de médicos especialistas indicada pelo perfil socioepidemiológico da população, em consonância com os princípios e as diretrizes do Sistema Único de Saúde - SUS. (BRASIL, 2011). 
No Brasil, a residência médica é considerada pós-graduação que confere ao concluinte título de especialista, regida por leis e regulamentos específicos, sendo exercida apenas em estabelecimentos autorizados pelo Ministério da Educação (NACUL et al., 2015). A Lei 6932 de 1981 rege os programas brasileiros de residência médica:

Art. $1^{\circ}$ - A Residência Médica constitui modalidade de ensino de pós-graduação, destinada a médicos, sob a forma de cursos de especialização, caracterizada por treinamento em serviço, funcionando sob a responsabilidade de instituições de saúde, universitárias ou não, sob a orientação de profissionais médicos de elevada qualificação ética e profissional.

$\int 1^{\mathrm{o}}$ - As instituições de saúde de que trata este artigo somente poderão oferecer programas de Residência Médica depois de credenciadas pela Comissão Nacional de Residência Médica.

$\int 2^{\circ}$ - É vedado o uso da expressão residência médica para designar qualquer programa de treinamento médico que não tenha sido aprovado pela Comissão Nacional de Residência Médica.

\30 A Residência Médica constitui modalidade de certificação das especialidades médicas no Brasil. [...] (BRASIL, 1981).

Nosso principal interesse está na Resolução 48 de 22 de junho de 2018, que atualizou a matriz de competência dos Programas de Residência Médica em Cirurgia Geral e do Programa de pré-requisito em Área Cirúrgica Básica no Brasil. Em síntese, destacamos:

Art. $2^{\circ} \mathrm{O}$ programa de residência médica em Cirurgia Geral terá duração de 3 (três) anos. Art. $3^{\circ}$ A conclusão do Programa de Pré-requisito em Cirúrgica Básica é condição indispensável para o ingresso nas especialidades cirúrgicas, que incluem: Cirurgia do Aparelho Digestivo, Cirurgia de Cabeça e Pescoço, Cirurgia Plástica, Cirurgia Vascular, Urologia, Cirurgia Torácica, Cirurgia Oncológica, Cirurgia Pediátrica e Cirurgia Coloproctológica.

$\int 1^{\circ}$ O Programa de Pré-requisito é constituído pelos Ciclos R1 e R2 constantes da Matriz de Competências em Cirurgia Geral anexa;

$\int 2^{\circ}$ A conclusão do Programa de Pré-requisito não confere título de especialista, conferindo ao concluinte um certificado que comprova sua competência para a atuação nos procedimentos cirúrgicos básicos listados no anexo.

$\int 3^{\circ}$ A certificação referida no parágrafo anterior será aceita para fins de aproveitamento em programas de residência médica de outras especialidades compatíveis, por prazo não superior a cinco anos, contados da emissão do certificado.

Art. $4^{\circ}$ A aplicação da Matriz de Competências no âmbito dos Programas de Residência Médica em Cirurgia Geral credenciados pela CNRM é obrigatória a partir do ano letivo de 2020. (BRASIL, 2018).

Ainda que a residência médica seja amparada por legislação específica, as questões inerentes aos processos de ensino-aprendizagem que ocorrem no seu cotidiano, não estão integralmente engessadas por caráter legislativo. Nos referimos, não à descrição de habilidades e competências necessárias ao médico cirurgião, mas aos modos como essas são desenvolvidas e adquiridas pelos residentes. 
Se, por um lado, a Residência em Cirurgia Geral produz experiências que envolvem o domínio de técnicas e de recursos tecnológicos, por outro, fatores intervenientes influenciam todo o período de especialização. As demandas da saúde pública, carências de insumos, de recursos humanos e infraestrutura, de relacionamento interpessoal etc, coabitam com exigências inerentes ao ensino. De sorte que cabe a preceptores e discentes estabelecer modos estritamente objetivos para o domínio de conteúdos e dos protocolos inerentes à prática cirúrgica. Eis, portanto, a necessária discussão sobre aquisição de habilidades e competências em Cirurgia Geral.

\section{Problematizando o Ensino de Habilidades e Competências}

De que trata o ensino ministrado na Residência Médica em Cirurgia Geral? Essa questão, norteadora da pesquisa implementada em primeira fase da investigação acerca da existência de materiais didáticos específicos para essa formação, foi tratada por revisão integrativa, como apontado anteriormente. A apresentação dos resultados prescindiu do uso de tabelas visando, tão somente, ilustrar nosso estudo, permitindo melhor conhecer a base da qual emergiram as inquietações que conduziram à elaboração do produto educacional.

a) Na base de dados Scielo: aplicando-se os descritores "Materiais de Ensino" AND "Internato e Residência" foi encontrado 01 artigo do ano de 2014. O mesmo não atendia aos critérios de inclusão e objetivos do estudo;

Com os descritores "Internato e Residência" AND "Cirurgia Geral" foram encontrados 12 artigos, dentre eles, 08 artigos atendiam aos critérios de inclusão por tratarem de Cirurgia Geral. Contudo, nenhum desses artigos tratavam de materiais de ensino para médicos-residentes em cirurgia geral.

Com os descritores "Ensino" $A N D$ "Internato e Residência" $A N D$ "Cirurgia geral” foram encontrados 05 artigos, sendo que todos eles eram repetidos;

Com os descritores "Ensino" $A N D$ "Materiais de Ensino" $A N D$ "Internato e Residência" foi encontrado 01 artigo, também repetido;

b) $\mathrm{Na}$ base de dados PUBMED - Com os descritores "Ensino" $A N D$ "Materiais de Ensino" $A N D$ "Cirurgia Geral" $A N D$ "Internato e Residência" não foram encontrados resultados;

Com os descritores "Teaching" $A N D$ "Teaching Materials" $A N D$ "General Surgery" $A N D$ "Intership and Residency" foram encontrados trabalhos significativos. Contudo, como na pesquisa desenvolvida na plataforma Scielo não foram encontrados trabalhos que tivessem em seu escopo apresentação, discussão, análise de produto educacional ou de ensino, material didático-pedagógico ou ainda, tecnologia inovadora no ensino de Cirurgia Geral. Os resultados encontrados foram: 2 estudos tratavam de ortopedia, 1 de anestesiologia, 1 de cirurgia vascular, 1 de cirurgia cardiovascular e 1 sobre o perfil profissiográfico de graduandos em medicina.

Sobre a Residência Médica em Cirurgia Geral, foram encontrados 3 artigos que tratavam do treinamento em simuladores para aperfeiçoamento de habilidades em laparoscopia, 1 sobre a substituição de valva mitral, 1 de habilidades de comunicação, 1 de preferência por estilos de aprendizagem, 2 de cateterização venosa central, 1 de proposição de checklist para avaliação de desempenho em simulador e outro de teste cognitivo em residentes de cirurgia geral, mas nenhum deles, como já registrado anteriormente, apresentou algum abordagem sobre o desenvolvimento de algum material com vistas a otimizar o desenvolvimento de habilidades e competências dos residentes.

Diante do exposto, chegou-se à conclusão de que há escassez de estudos que tratam de materiais didático-pedagógicos implementados para o desenvolvimento de competências e 
habilidades dos discentes em Residência Médica em Cirurgia Geral, inferindo-se desta evidência a necessidade de se pensar a elaboração de produto educacional no intuito de responder a essa lacuna.

Contudo, qual a melhor forma de produzir domínio e aplicação dos conteúdos inerentes a essa especialização? Essa não é uma questão tão simples de ser respondida; afinal, são muitas as teorias com pretensões de se validar a forma considerada a mais acertada para se conduzir a relação ensino-aprendizagem.

A escolha por uma teoria de aprendizagem deve levar em conta especificidades relativas ao processo educativo almejado. Tendo por base o conhecimento pregresso dos residentes, adquirido no contexto da graduação em medicina, em que uma série de conceitos foram tratados de modo genérico e que, portanto, atuariam como subsunçores às complexas análises que são desenvolvidas na especialização em Cirurgia Geral, adotou-se a teoria da Aprendizagem Significativa de Ausubel (1918-2008) como base da sequência didática projetada para o material didático-pedagógico intitulado Guia do Residente em Cirurgia Geral.

Essa teoria, tem por base os estudos desenvolvidos por Ausubel, acerca da psicologia da aprendizagem, e se aplica de modo adequado à proposta que estabelecemos, uma vez que a aquisição de nova informação, acerca das técnicas e processos cirúrgicos, deve se tornar duradoura e flexível, como afirmam (ARAÚJO e MENEZES, 2019.p.14):

\footnotetext{
Para que a aprendizagem significativa ocorra, três requisitos devem ser cumpridos. Em primeiro lugar, o material a ser aprendido em si deve ter potencial significado (...). Em segundo, o aluno deve possuir conceitos e proposições relevantes, que servirão para ancorar a nova aprendizagem e assimilar novas ideias. Em terceiro lugar, ele deve escolher relacionar a nova informação para a sua estrutura cognitiva de forma não literal. Se qualquer um desses três elementos é escasso, a aprendizagem significativa não pode ocorrer, pelo menos nos estágios iniciais de uma determinada sequência de aprendizagem.
}

Essa retenção de novo conteúdo seria considerada duradoura, pois não ocorreria sua perda com rapidez e mesmo que ela venha ocorrer ao longo do tempo, seria rapidamente relembrada através de um simples novo contato com o conteúdo esquecido. Flexível significa dizer que seria realmente compreendida pela pessoa, e não apenas memorizada; e, portanto, de posse da informação obtida, de maneira significativa, o aprendiz se tornaria capaz de aplicá-la em diversas situações e formatos, além de ser capaz de explicá-la para outras pessoas, inclusive de forma modificada (MOREIRA, 1999).

Para Ausubel a retenção das informações no cérebro humano é realizada de forma organizada e seguindo uma hierarquia conceitual, na qual elementos mais específicos são ligados a elementos mais abrangentes, mais inclusivos. A aquisição de uma informação se faz de forma 
significativa se ela transcorre por intermédio de um processo de relação, ou melhor, através do ancoramento e fusão de uma nova informação a um conhecimento prévio que o aprendiz possui e que está localizado na área cognitiva. Esta informação prévia (conhecimento, símbolo, conceito proposição, modelo mental, imagem) presente na estrutura cognitiva e que se relaciona com a nova informação é definida por Ausubel como subsunçor (MOREIRA, 1999).

Então, subsunçor é o nome que se dá a um conhecimento específico, existente na estrutura de conhecimentos do indivíduo, que permite dar significado a um novo conhecimento que lhe é apresentado ou por ele descoberto (MOREIRA, 1999). Para que haja algo novo aprendido, são necessárias a organização e a interação do novo material a essa estrutura cognitiva.

O conteúdo que é novo elemento cognitivo é aprendido à medida que encontra um ponto de ancoragem - os subsunçores - na estrutura cognitiva já estabelecida no indivíduo. Os subsunçores são conceitos e ideias relevantes, previamente organizadas, claras e apropriadas. Quando o sujeito está diante de algo novo e potencialmente significativo, se houver uma interação entre o novo conteúdo e o conteúdo prévio já existente em sua mente, aumenta-se a probabilidade de ocorrer a aprendizagem (AUSUBEL, 2003).

\section{Proposta de Material de Ensino: Guia do Residente em Cirurgia Geral}

Para a otimização do ensino em cirurgia geral, optou-se pela elaboração de um guia que possa ser usado pelo médico-residente. Compreendendo o material proposto como uma ferramenta que objetiva amparar o processo de ensino-aprendizagem do discente, identificou-se a necessidade de estruturar o Guia de modo que a apreensão de conteúdos novos fosse facilitada pelos conteúdos estudados anteriormente.

Além disso, o escopo do material denominado Guia do Residente em Cirurgia Geral foi elaborado de acordo com ordenamento lógico subjacente à Teoria da Aprendizagem Significativa, de Ausubel, apresentando, quanto à complexidade dos conteúdos, ordem gradativa e progressiva e, quanto ao método de ensino, a sequência didática. Em síntese, a disposição dos temas tratados pelo Guia, ordenou de forma conectiva possíveis subsunçores a novos conceitos relativos aos objetivos do ensino em Cirurgia Geral (Quadro 1). 


\section{Dialogia}

CARVALHO, Igor Pereira de; PEREIRA, Adilson; OLIVEIRA, Ivanete da Rosa Silva de. Ensino do médico residente em cirurgia geral: proposta de ferramenta didático-pedagógica

Quadro 1 - Organização da sequência de temas do guia

\begin{tabular}{|c|c|}
\hline 01 & $\begin{array}{l}\text { Introdução } \\
\text { Residência em Cirurgia Geral: Definição, aspecto histórico, abordagens de temas segundo diretrizes } \\
\text { curriculares, o ensino em Cirurgia geral }\end{array}$ \\
\hline 02 & $\begin{array}{l}\text { Paramentação cirúrgica } \\
\text { O que é? } \\
\text { Como fazer? } \\
\text { Quais os componentes? }\end{array}$ \\
\hline 03 & $\begin{array}{l}\text { Instrumentação Cirúrgica } \\
\text { O que é? } \\
\text { Quais os componentes? }\end{array}$ \\
\hline 04 & $\begin{array}{l}\text { Avaliação pré-anestésica } \\
\text { O que é? } \\
\text { Qual a sua importância? }\end{array}$ \\
\hline 05 & $\begin{array}{l}\text { Anestesia Local } \\
\text { Materiais necessários } \\
\text { Como fazer? }\end{array}$ \\
\hline 06 & $\begin{array}{l}\text { Sutura } \\
\text { Materiais necessários } \\
\text { Como fazer? }\end{array}$ \\
\hline 07 & $\begin{array}{l}\text { Drenagens } \\
\text { Tipos } \\
\text { Materiais necessários } \\
\text { Como fazer? }\end{array}$ \\
\hline 08 & $\begin{array}{l}\text { Acesso venoso profundo } \\
\text { Locais de acesso } \\
\text { Materiais necessários } \\
\text { Como fazer? }\end{array}$ \\
\hline 09 & $\begin{array}{l}\text { Cricotireoidostomia } \\
\text { Materiais necessários } \\
\text { Como fazer? }\end{array}$ \\
\hline 10 & $\begin{array}{l}\text { Traqueostomia } \\
\text { Materiais necessários } \\
\text { Como fazer? }\end{array}$ \\
\hline
\end{tabular}

Fonte: Elaboração dos autores (2020).

Em síntese, o Guia do Residente em Cirurgia Geral, visa facilitar o trabalho de preceptores acerca do conteúdo ministrado a médicos-residentes, de modo que possam recorrer a material didático-pedagógico claro e objetivo, em função do domínio de técnicas e as necessárias habilidades 
envolvidas nas atividades práticas em cirurgia geral. A disponibilidade do produto, em sua versão final, estará na plataforma EDUCAPES, no segundo semestre do ano de 2021, consignada sua apresentação em banca de defesa no programa de Pós-graduação Stricto Sensu em Ensino em Ciências da Saúde e Meio Ambiente. Uma terceira fase da pesquisa está planejada para ocorrer em período egresso ao mestrado, quando o Guia do Residente em Cirurgia Geral será implementado em aplicativo para plataforma móvel, consignando-se adaptação à necessária inovação na educação médica.

\section{Método}

Para avaliar o Guia foi produzida pesquisa de campo, com abordagem descritiva e exploratória, de natureza qualitativa. Justifica-se a opção por essa modalidade de pesquisa pelo fato de a temática exigir experiência e contato próximo do participante ao público-alvo, isto é, médicosresidentes, possibilitando interpretações, conclusões e conhecimento da realidade do objeto de estudo.

O método adotado foi o empírico indutivo, tendo como técnica de entrevista semiestruturada (TRIVIÑOS,1987), tendo como sujeito participante/avaliador médico cirurgião e preceptor que atua em programa de residência médica em cirurgia geral. O entrevistado assinou o Termo de Consentimento Livre e Esclarecido (TCLE), concordando em participar da pesquisa.

Para a validação prévia do protótipo, em projeto piloto, foi enviado ao participante o material 30 dias antes da referida entrevista para análise preliminar. Registramos, contudo, que devido a pandemia COVID-19, o participante aceitou os critérios de ser entrevistado por via remota, utilizando-se o aplicativo whatsapp ${ }^{\circledR}$.

A ampliação de participantes no processo de validação do produto educacional sofreu o interdito desse período de pandemia, devido às demandas hospitalares e seus restritos acessos. Até o momento que os dados foram analisados, as medidas restritivas estavam em curso. Contudo, as respostas obtidas com a aplicação do roteiro de entrevista ao especialista atuante na área foram analisadas e consideradas válidas para a pesquisa.

\section{Apresentação e análise dos resultados}

Embasando-se na entrevista do participante, foi descrito que durante sua carreira profissional deparou-se com contextos nos quais apresentou dificuldades quanto ao processo de ensino-aprendizagem dos discentes, devido, principalmente, a ausência de material didático que pudesse otimizar o aprendizado. 
A aprendizagem envolve a capacidade de tornar o indivíduo capaz, mediante domínio de certo conteúdo, por meio de estudo, observação, experimentação, que lhe possibilite compreensão e retenção do conteúdo e da habilidade psicomotora atrelada ao mesmo. No caso das competências em cirurgia geral, "a habilidade é uma resposta comportamental na prática estabelecida pela repetição frequente e continuada de movimentos que ampliam a agilidade, a destreza, a precisão no manejo e na percepção sensitivo-motora. (COSTA et al., 2018, p. 3)

O contexto de domínio das habilidades não está separado do desenvolvimento da aptidão e da proficiência, muito embora ambas sejam distintas. No caso da Cirurgia Geral, o médico é apto, quando ele desenvolve, após o treinamento, o "ponto máximo da percepção sensitivomotora, enquanto a proficiência é quando a aptidão, competência e retenção de habilidade atingem uma estabilidade, um platô", expressando-se como pleno domínio de técnicas e conhecimento. (COSTA et al., 2018, p. 3).

Os principais pontos destacados pela entrevista acerca do protótipo do material didáticopedagógico associado ao projeto piloto de validação inicial do produto educacional, foram:

- Sugestão de acréscimo novos temas. Dentre eles, instrumentação cirúrgica, acesso venoso periférico, cateterização e drenagens;

- Otimização do tempo de estudo do aluno, sugerindo texto sintético, objetivo e intuitivo, com o intuito de tornar célere e otimizado o processo de ensino-aprendizagem do discente;

- Sugestão de algumas obras literárias, afim de aumentar a robustez da ancoragem teórica e deixar a organização do guia mais fluída e intuitiva;

- Refere escassez de material didático-pedagógico que auxilie e seja focado no ensino de médicos residentes em cirurgia geral;

- Destaca habilidades motoras, raciocínio clínico-cirúrgico e capacidade de resiliência para adaptação em diversos cenários que a prática profissional exige.

Segundo Costa et al (2018), para a aprendizagem de habilidades psicomotoras em cirurgia geral ser efetivada, se faz necessária a presença de fatores como a maneira que é estruturada a prática, o cenário em questão, a interação docente e discente e artifícios pedagógicos.

Em síntese, a entrevista apontou a questão da otimização do tempo de dedicação ao estudo pelo discente como um dos mais significativos fatores considerados. Segundo Torres (2018), a residência médica é um período imersivo em uma determinada especialidade. Com isso, a otimização do tempo em relação ao estudo teórico traz consigo ganho em qualidade de vida para o discente, com a possibilidade de usufruir de horas de sono e práticas de lazer. 
O material didático-pedagógico avaliado foi considerado adequado na facilitação do aprendizado de conceitos, propiciando conexões lógicas e duradouras, como apontamos com o uso da teoria de aprendizagem de Ausubel em sua estruturação. Esse fator produz melhor uso do tempo investido pelo discente em relação ao estudo e domínio de conceitos. Sabe-se que muitos materiais didático-pedagógicos são expressos de modo prolixo e o tempo dispensado à sua decodificação é precioso para os discentes Residentes em Cirurgia Geral, já assoberbados em tarefas que exigem foco, precisão técnica e domínio de habilidades cognitivas e motoras.

O fato de se produzir material didático-pedagógico sob a forma de Guia para o Residente em Cirurgia Geral, não resolve todos os problemas relativos à facilitação da aprendizagem nessa especialização, mas é uma ferramenta auxiliar, cujos impactos podem ser relevantes na minimização do estresse que esses discentes enfrentam.

Os residentes vivenciam outros problemas que merecem estudos aprofundados, como a prevenção da síndrome de Burnout e do suicídio em médicos residentes de cirurgia geral, como alerta o trabalho de Soares et al. (2012), "Burnout e pensamentos suicidas em médicos residentes de hospital universitário". Não sendo este o objeto do qual nos ocupamos, ao menos, facilitar o aprendizado dos residentes em Cirurgia Geral, já seria fator potencializador do prazer que se pode obter com o processo de aprendizagem.

\section{Considerações finais}

A Residência Médica em Cirurgia Geral ocorre em período de duração de dois anos, para se obter domínio de competências da área básica em cirurgia geral e três anos para se obter a especialização em cirurgia geral. Contudo, é fato que essa especialidade não possui material didático pedagógico padronizado pelo Ministério da Educação, nem pelo Colégio Brasileiro de Cirurgiões, para se estabelecer alguma padronização na operacionalização das práticas cirúrgicas.

Contudo, não sendo foco de nossa pesquisa discutir se haveria essa obrigatoriedade institucional, ao menos, apontamos, por revisão integrativa produzida em primeira fase de nossa investigação, a existência de lacunas relativas a publicações sobre o desenvolvimento de materiais de ensino em Cirurgia Geral. Assim, elaborou-se o Guia do Residente em Cirurgia Geral, procurando-se otimizar a aprendizagem dos conteúdos e o desenvolvimento de habilidades e competências inerentes às práticas cirúrgicas.

Sua validação inicial, ainda que sob os interditos e limitações advindas das medidas restritivas e demandas ocasionadas pela pandemia de COVID-19, permitiu verificar a pertinência do referido material e sua necessidade como ferramenta auxiliar para o uso de preceptores e 
discentes dessa especialização. Como mencionado, o uso do Guia é ferramental didático. Seu bom uso dependerá do modo como preceptores e discentes o operacionalizam.

Todavia, acredita-se que em uma revisão integrativa vindoura, esse material tenha incentivado a elaboração de outros, de forma que ferramentas dessa modalidade sejam multiplicadas e disseminadas junto aos programas de Residência Médica em Cirurgia Geral, possibilitando inovações nos modos de ensino dessa área.

\section{Referências}

ARAUJO, Liércio A.; MENEZES, Robson Lúcio silva. EDUCAÇÃO E APRENDIZAGEM: A TEORIA DA APRENDIZAGEM SIGNIFICATIVA EM DAVID AUSUBEL. REVASF, Petrolina- Pernambuco - Brasil, vol. 9, n.19, p. 04-25, agosto, 2019. Disponível em: https://www.periodicos.univasf.edu.br/index.php/revasf/article/download/468/308/1443. Acesso em 19 de julho de 2021.

AUSUBEL, D. P. Aquisição e retenção de conhecimentos: uma perspectiva cognitiva. Trad. Ligia Teopisto. Lisboa: Plátano Edições Técnicas, 2003. Tradução de The acquisition and retention of knowledge: a cognitive view.

BRASIL. Decreto n ${ }^{\circ} 80281$, de 05 de setembro de 1977. Regulamenta a Residência Médica, cria a Comissão Nacional de Residência Médica e dá outras providências. Diário Oficial da União. Disponível em: Microsoft Word - d80281.doc (mec.gov.br). Acesso em 13 dez 2020.

BRASIL. Decreto $n^{\circ} 7562$, de 15 de setembro de 2011. Dispõe sobre a Comissão Nacional de Residência Médica e o exercício das funções de regulação, supervisão e avaliação de instituições que ofertam residência médica e de programas de residência médica. Diário Oficial da União. Disponível em: 1(Pag) (mec.gov.br). Acesso em 13 dez 2020.

BRASIL. Lei n ${ }^{\circ}$ 6932, de 07 de julho de 1981. Dispõe sobre as atividades do médico residente e dá outras providências. Diário Oficial da União. Disponível em: < http://www.planalto.gov.br/ccivil_03/Leis/L6932.htm . Acesso em 02 out 2020.

BRASIL. Ministério da Educação. Secretaria de Educação Superior. Resolução n ${ }^{\circ}$ 48, de 28 de junho de 2018. Dispõe sobre a Matriz de Competências dos Programas de Residência Médica em Cirurgia Geral e do Programa de Pré-requisito em Área Cirúrgica Básica no Brasil. Diário Oficial da União. Disponível em: < RESOLUÇẪO No 48, DE 28 DE JUNHO DE 2018 - Imprensa Nacional (in.gov.br) $>$. Acesso em 13 dez 2020.

COSTA, Grijalva Otávio Ferreira da et al. Taxonomia dos objetivos educacionais e as teorias de aprendizagem no treinamento das técnicas cirúrgicas laparoscópicas em ambiente de simulação. Rev. Col. Bras. Cir., Rio de Janeiro, v. 45, n. 5, e1954, 2018. Available from http:/ / www.scielo.br/scielo.php?script=sci_arttext\&pid=S010069912018000500400\&lng=en\&nrm=iso. access on 31 Oct. 2020. Epub Oct 18, 2018. http://dx.doi.org/10.1590/0100-6991e-20181954. 
MOREIRA, A. M. A teoria da Aprendizagem significativa de Ausubel. In: MOREIRA, A. M. Teorias de Aprendizagem. EPU: São Paulo, 1999. 151-165p. Disponível em: AusubelMoreira.pdf (usp.br). Acesso em: 13 dez. 2020.

NACUL, Miguel Prestes; CAVAZZOLA, Leandro Totti; MELO, Marco Cezário de. Situação atual do treinamento de médicos residentes em videocirurgia no Brasil: uma análise crítica. ABCD, arq. bras. cir. dig., São Paulo, v. 28, n. 1, p. 81-85, 2015. Available from http:/ / www.scielo.br/scielo.php?script=sci_arttext\&pid=S0102$67202015000100081 \& \operatorname{lng}=$ en\&nrm $=$ iso. access on 30 Oct. 2020. https://doi.org/10.1590/s0102-67202015000100020.

SANTOS, Elizabeth Gomes dos. Residência médica em cirurgia geral no Brasil - muito distante da realidade profissional. Rev. Col. Bras. Cir., Rio de Janeiro, v. 36, n. 3, p. 271-

276, jul. 2009. Disponível em http://www.scielo.br/scielo.php?script=sci_arttext\&pid=S010069912009000300017\&lng=pt\&nrm=iso. acessos em 30 out. 2020. http://dx.doi.org/10.1590/S0100-69912009000300017.

Simpson E. The classification of educational objectives in the psychomotor domain: The psychomotor domain. Washington, DC: Gryphon House; 1972.

SOARES, Leonardo Ribeiro et al. Burnout e pensamentos suicidas em médicos residentes de hospital universitário. Rev. bras. educ. med., Rio de Janeiro, v. 36, n. 1, p. 77 -

82, Mar. 2012. Available from http://www.scielo.br/scielo.php?script=sci_arttext\&pid=S0100$55022012000100011 \& \operatorname{lng}=$ en\&nrm=iso. access

on 15 Dec. 2020. https://doi.org/10.1590/S0100-55022012000100011.

SOUZA, Marcela Tavares de; SILVA, Michelly Dias da; CARVALHO, Rachel de. Integrative review: what is it? How to do it?. Einstein (São Paulo), São Paulo, v. 8, n. 1, p. 102-

106, Mar. 2010. Available from

http:/ / www.scielo.br/scielo.php?script=sci_arttext\&pid=S1679-

45082010000100102\&lng $=$ en\&nrm $=$ iso. access

on 12 Dec. 2020. https://doi.org/10.1590/s1679-45082010rw1134.

TONATTO FILHO, Antoninho José et al. O Reflexo do Aumento de Vagas da Residência de Cirurgia Geral no Brasil. Rev. bras. educ. med., Brasília, v. 44, n. 1, e007, 2020. Available from http://www.scielo.br/scielo.php?script=sci_arttext\&pid=S0100-

55022020000100213\&lng=en\&nrm=iso. access on 30 Oct. 2020. Epub Mar 13, 2020. http://dx.doi.org/10.1590/1981-5271v44.1-20190178.ing.

TORRES, R. A. T. Gestão do tempo no cotidiano de médicos residentes de Clínica Médica em um hospital público universitário de São Paulo, Brasil. Dissertação (Mestrado em Ciências) - Faculdade de Saúde Pública, Universidade de São Paulo. São Paulo, p. 123. 2018. Disponível em: < Microsoft Word FINAL.doc (usp.br) >. Acesso em 15 dez 2020.

TRIVIÑOS, A. N. S. Introdução à pesquisa em ciências sociais: a pesquisa qualitativa em educação. São Paulo: Atlas, 1987. 